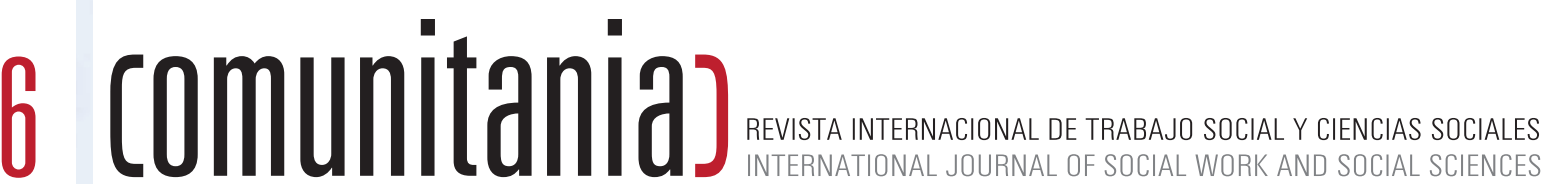

IGNASI BRUNET ICART | JOAN RODRÍGUEZ SOLER | BEATRIZ ESCUDERO BERZAL | PALOMA SERRANO POSTIGO LUIS MANUEL RODRÍGUEZ OTERO | MARTHA LETICIA CABELLO GARZA | MARÍA CONCEPCIÓN ARROYO RUEDA 


\title{
Aproximación al estado de bienestar en Polonia Aproximation to the welfare state in Poland
}

\author{
Paloma Serrano Postigo* \\ * secretariaacademica@segovia.uned.es
}

\begin{abstract}
Abstrac:
This article analyses the transformation of the Welfare State in Poland from 1945 to the present day. In order to achieve this objective, after briefly outlining the genesis, evolution and development of the Welfare State in Europe, this article explains the Welfare system in Poland since the communist era, its development during the Polish transition period to the system of democracy today and the consequences of the historical of joining the EE in 2204.
\end{abstract}

Keywords: Poland, Welfare State, social cohesion, health care, European Union, communist bloc.

\section{Resumen:}

El presente artículo aborda el proceso de transformación del Estado de Bienestar en Polonia desde 1945 hasta la actualidad. Para ello, y tras exponer de forma breve cuál ha sido la génesis, evolución y desarrollo del Estado de Bienestar en el continente europeo, se analiza a continuación el Estado de Bienestar en Polonia desde la etapa comunista, su evolución durante la transición polaca hacia el sistema de democracia parlamentaria y economía de mercado para concluir en el momento actual teniendo en cuenta el hecho histórico del ingreso de Polonia en la Unión Europea en el año 2004.

Palabras clave: Polonia, Estado de Bienestar, cohesión social, protección de la salud, Unión Europea.

\section{Article info:}

Received: 08/05/2013 / Received in revised form: 12/06/2013

Aceppted: 01/07/2013 / Published online: 15/07/2013

DOI: http://dx.doi.org/10.5944/comunitania.6.3/

\section{El Estado de Bienestar: Génesis, evolución y desarrollo}

Puede afirmarse que la consolidación teórica y práctica del Estado de Bienestar se produjo tras la Segunda Guerra Mundial gracias, en parte, a la puesta en práctica de 
los postulados keynesianos. Las críticas que realizó John Maynard Keynes (18331946) en sus escritos, dirigidas al modelo de mercado basado en el liberalismo económico imperante durante la primera preguerra, supusieron el punto de partida para que el modelo de Estado de Bienestar, tal y como se entiende en la actualidad, tomara carta de naturaleza.

En su obra, Las consecuencias económicas de la paz, escrita tras la Primera Guerra Mundial, el citado autor pone de relieve cómo el funcionamiento de la mayoría de los sistemas económicos capitalistas han errado al intentar acentuar el ahorro frente al consumo en sociedades donde, junto a las clases adineradas, existe una clase trabajadora dispuesta a no renunciar a sus pequeños privilegios. En este sentido, “las clases trabajadoras pueden no querer seguir por más tiempo en tan amplia renuncia, y las clases capitalistas, perdida la confianza en el porvenir, pueden tener la pretensión de gozar más plenamente de sus facilidades para consumir mientras ellas duren, $y$, de este modo, precipitar la hora de su confiscación" (Keynes 1991: 20).

Una mayor consumo junto a una mayor demanda de inversión, son a juicio de Keynes, los dos elementos necesarios para que las economías capitalistas se reactiven. Pero para que la reactivación cobre sus frutos es necesaria la intervención del Estado. El Estado debe intervenir para poder garantizar la aprobación de leyes sociales que mejoren las condiciones de vida de los trabajadores en activo así como de las clases menos favorecidas. Asimismo, la citada intervención del Estado puede promover programas de obras públicas que reactiven la inversión.

Pero las expresadas ideas keynesianas solo supusieron la consolidación del Estado de Bienestar. Previamente a las mismas, en la Alemania de Bismarck la aprobación de un conjunto de leyes pioneras relacionadas con la salud marcaron un antes y un después en la historia de aquel. Coetáneas a las reformas del Canciller alemán, las políticas sociales británicas de finales del siglo XIX se tradujeron en la promulgación de un elenco de leyes. Sin embargo, no sería hasta finales de la Segunda Guerra Mundial, cuando se asienten los cimientos del Estado de Bienestar gracias, principalmente, al trabajo llevado a cabo por el Barón de Tugall, William Henry Beveridge (1879-1963) a través de la publicación de los llamados Informes Beveridge, encargo del que fuera entonces ministro de Trabajo, Ernest Bevin. El primero (Social Insurance and Allied Services) vio la luz en noviembre 1942; el segundo (Full Employment in a Free Society) lo hizo en 1944.

Con los antecedentes señalados, el Estado de Bienestar conocerá su "época dorada" en el continente europeo durante las décadas de 1950 y 1960. A partir de la década siguiente, el Estado de Bienestar comienza a entrar en crisis por el agotamiento del modelo keynesiano arrastrado, in fine, por las corrientes neoliberales que surgen a finales de 1970. Los últimos veinte años del siglo XX traerán consigo una serie de cambios en todos los órdenes. Conceptos como "globalización", "mundialización", "gobernanza mundial" o"transnacionalismo", transformarán la forma de entender 
no sólo las relaciones económico-sociales y políticas dentro y fuera de los Estados sino también las relaciones entre los individuos. Todo ello aderezado por la revolución en las tecnologías de la información.

El Estado de Bienestar configurado a partir de tres hechos básicos, crecimiento económico, principio de legitimidad del Estado y universalismo de los derechos sociales deberá evolucionar para poder adaptarse a las nuevas circunstancias, a saber: transformaciones en el trabajo, en el modelo de familia y en las comunidades. El siglo XXI, se presenta, pues como un siglo de cambios, cambios que acontecen de manera vertiginosa al socaire de la revolución tecnológica. Queda claro, que dichos cambios, también afectarán al modo de entender y desarrollarse el Estado de Bienestar. La evolución de la sociedad y las variaciones en el modelo económico hacen que aquel tenga que reformularse de manera continua en una "vida líquida" tal y como define Zygmunt Bauman a la vida actual donde las propias personas se convierten en objeto de consumo. Dice el citado autor: "La vida líquida es una vida devoradora. Asigna al mundo, a las personas y a todos sus demás fragmento animados e inanimados el papel de objetos de consumo: es decir, objetos que pierden su utilidad ( $y$, por consiguiente, su lustre, su atracción, su poder seductivo y su valor) en el transcurso mismo del acto de ser usados. Condiciona además, el juicio y la evaluación de todos estos fragmentos según el patrón de objetos de consumo)" (Bauman 2007: 51).

\section{Diferentes modelos del Estado de bienestar}

Partiendo de que el Estado de Bienestar se puede definir como un Estado tal donde el poder institucionalizado es utilizado de forma deliberada (por la política y la administración) en un esfuerzo por modificar el juego de fuerzas del mercado (Fernández-García y López Peláez 2006: 36), la implantación definitiva de aquel en el continente europeo tras la Segunda Guerra Mundial, no trajo consigo un mismo modelo de Estado de Bienestar.

La redefinición de las relaciones políticas entre los países pertenecientes a los llamados "bloque capitalista" y bloque comunista" -con EEUU y la URSS ocupando el lugar de superpotencia en cada uno de éstos- determinó, en gran medida, el modelo económico-social a seguir. Mientras que los países pertenecientes al “bloque capitalista" desarrollaron un modelo económico basado en la economía de mercado, los países englobados en el "bloque comunista" quedaron condicionados por un sistema diseñado desde la URSS pero con pequeños matices en cada uno de los países satélites, sistema basado en una economía planificada.

Derivado de lo expresado en el párrafo anterior, la concepción del modelo de Estado en ambos bloques fue totalmente antagónica. Mientras que en Europa occidental, al Estado se le fue añadiendo diferentes calificativos como Estado social y democrático de Derecho y/o Estado de Bienestar dentro del sistema jurídico-político de la 
democracia parlamentaria ${ }^{1}$, en la Europa del Este, el Estado quedó engranado a las llamadas "democracias populares" a través de una construcción historicista pero también filosófica derivada de los postulados marxistas-leninistas que abogaban, a priori, por la no existencia del Estado en la lucha de la clase proletaria frente a la clase burguesa. Cuando el Estado se convierta, finalmente, en representante efectivo de toda la sociedad, será por sí mismo superfluo (Engels 1976: 51). Lo cierto es que, la deriva del Estado en los países comunistas no supuso la desaparición del mismo, sino la consolidación de éste como máximo proveedor de las necesidades básicas del conjunto de la sociedad: educación, vivienda y sanidad.

Debe entenderse que dicha provisión del Estado respondía a la idea de la creación y consolidación del sistema socialista a través de la transformación revolucionaria de las estructuras económicas y sociales para alcanzar la igualdad de oportunidades desde una acción colectiva. El Welfare State tal y como fue concebido en Europa occidental nada tuvo que ver con el Estado de Bienestar de los países comunistas.

Partiendo de los postulados generales que acabamos de definir, la evolución ideológica del Estado de Bienestar desde el punto de vista de las teorías marxistas ha servido para desarrollar un neomarxismo que concibe a éste como un Estado que asegura y representa la propiedad privada y que calcula recompensas sociales y políticas a la clase trabajadora a través de los sistemas de bienestar, fundamentalmente de servicios sociales. Además de preservar el status quo y el poder establecido, la política social, los programas de bienestar y de servicios sociales en materia de salud, educación y vivienda, etc...., servirían a los intereses del capital porque vendrían a incrementar la eficiencia económica y a asegurar la reproducción de las relaciones sociales del capital. (Garcés 2006: 117).

El estudio del Estado de Bienestar en Polonia desde la transición hasta la actualidad que se realizará en el apartado siguiente nos servirá para entender lo señalado en el párrafo precedente. Hay que tener en cuenta que, a pesar de que la república del águila blanca quedó integrada desde el final de la Segunda Guerra Mundial en el "bloque comunista", -y por tanto homogeneizada con el resto de los países satélites de la URSS en su devenir socioeconómico y político-, no es menos cierto que las peculiaridades de Polonia como Estado, peculiaridades referentes, entre otros factores a la necesidad de preservar la identidad colectiva marcada profundamente por el hecho religioso, servirá como elemento diferenciador a la hora de examinar el Estado de Bienestar.

1 Debe especificarse que el modelo de Estado de Bienestar en Europa occidental no ha correspondido desde el principio a un modelo único. Así, mientras países como Alemania, Francia, Bélgica y Holanda han optado por un Estado de Bienestar conservador o "bismarckiano", el modelo nórdico o socialdemócrata se encuentra ligado a la política keynesiana. España e Italia se considerarían pertenecientes a un tercer modelo, el sureño, basado en la familia. 


\section{El Estado de Bienestar en Polonia}

\subsection{Características del Estado de Bienestar durante la etapa socialista: 1945-1989}

Resultaría muy difícil entender cómo se desarrolló el Estado de Bienestar durante la transición polaca sin señalar de forma sucinta cuales fueron las características de aquel durante la etapa socialista. Retomando la idea expresada en la pregunta anterior, a pesar de que inicialmente la URSS previó la implantación uniforme de un sistema de economía planificada en todos los países integrantes del "bloque comunista", hay que decir que, la realización práctica del mismo no fue igual en todos los países satélites.

Las diferentes tradiciones culturales de cada país, su historia e historiografía, sus complejas sociedades así como sus diversos sistemas políticos y económicos resultaron ser, con el paso del tiempo, el "caballo de Troya" con el que tuvieron que enfrentarse los soviéticos a la hora de intentar mantener la uniformización y homogeneización del conjunto de países que quedaron bajo la égida comunista. Polonia no fue la excepción.

Dicho lo anterior, es cierto que, en una primera etapa la URSS consiguió extrapolar su modelo de economía planificada a los países satélites. La nacionalización de los principales sectores económicos, la necesidad imperiosa de la industrialización y la colectivización de la agricultura, fueron los instrumentos con los que contó el país soviético para asegurar el control económico, pero también político e ideológico de aquellos.

A medida que avanzaba el tiempo, las diferencias fueron surgiendo, a saber: Alemania del Este, la República Popular de Polonia, Bulgaria o Rumania, entre otros países pertenecientes al bloque, fueron mostrando sus propias singularidades. El proceso de colectivización en Polonia nada tuvo que ver con el proceso de colectivización de sus vecinos. De igual modo ocurrió con el proceso de nacionalización. Y algo similar ocurrió también con la política social aplicada a estos países. Como señala de manera proverbial Stanislawa Golinoska respecto a Polonia: "The social system of the Polish People Republic was not a typical model or a socialist state, and diverged widely from the social systems of Poland's neighbours; Czecoslovakia, Eastern Germany and the USSR". (Golinoska 2009: 222)

Enlazando con el párrafo anterior, las principales características del Estado de Bienestar polaco durante la etapa socialista pueden quedar resumidas en los siguientes puntos, a saber:

1. En primer lugar, no todos los trabajadores disfrutaron del mismo sistema social. Los trabajadores de la industria fueron los aventajados respecto a los campesinos que no vieron igualados la protección de sus derechos sociales con los primeros 
hasta la década de 1990. La construcción del "socialismo real" pasaba, entre otros objetivos, por desarrollar una rápida industrialización dando preferencia a los bienes de equipo frente a los bienes de consumo. El proletariado era esencial para alcanzar los objetivos económicos impuestos en cada plan. En cierto modo, asegurarles la cobertura de las necesidades básicas suponía un "premio" al trabajo desempeñado y una garantía del buen funcionamiento del sistema económico en su conjunto.

Con lo que respecta al campesinado cabe decir que, fueron las "cooperativas médicas" el medio a través del cual se diseño un sistema paralelo al estatal para cubrir las necesidades básicas de este sector de la población.

2. En segundo lugar, el sistema de asistencia en los tres pilares básicos, sanidad, educación y vivienda, no pudo ser garantizado en su totalidad por el Estado. El papel de la Iglesia y de la familia, basada en la tradición de valores y costumbres, fue esencial a la hora de cubrir aquellos. Los dirigentes de la República Popular permitieron que las organizaciones sociales provenientes de la Iglesia hicieran su papel, siempre bajo el estricto control y supervisión de los órganos gubernamentales diseñados y encargados de cumplir con dicha tarea. El nacimiento de asociaciones profesionales y sindicatos jugaron también un papel esencial. A pesar de su estrecho margen de maniobra y de libertad en su autoorganización supusieron un instrumento muy eficaz de enlace entre el Este y el Oeste además de servir de cauce para que la oposición al régimen pudiera tener una pequeña salida hacia el exterior tanto dentro del sistema comunista como fuera de él.

No obstante, debe tenerse en cuenta que, y respecto al sistema educativo, la estrategia del Partido Comunista Polaco, fue desde un primer momento, popularizar la educación mediante el acceso a las escuelas y a las universidades de jóvenes de origen obrero y campesinos, dificultando el mismo a las personas pertenecientes a las antiguas clases privilegiadas. El cambio en los programas de enseñanza mediante la introducción de manuales soviéticos respondió a la eliminación de los programas de filosofía y de sociología "burguesas" sustituidas por el marxismo. (Kieniewicz 2000: 225). De este modo, mediante la centralización de la educación se consiguió controlar la cultura y por ende, la ideología de todo el país.

3. El problema de la vivienda tras la destrucción de las grandes ciudades polacas -como Varsovia durante la Segunda Guerra Mundial-2, se convirtió en una de las prioridades del partido comunista polaco siempre por detrás del objetivo número uno, a saber: la rápida industrialización. En realidad, la primera iba ligada inexcusablemente al segundo. Los éxodos masivos del campesinado a la ciudad en busca de trabajo en las fábricas obligaron a los dirigentes polacos a elaborar un plan de

2 En Varsovia, el 85\% de las viviendas quedaron completamente destruidas. En el resto del país, la destrucción llegó a ser de un $50 \%$ en las ciudades y de un $40 \%$ en el ámbito rural. 
vivienda que fuera capaz de asumir la mano de obra tan necesaria para el desarrollo de la industria pesada. Grandes bloques de vivienda iguales con pequeños apartamentos construidos de acuerdo con el modelo de arquitectura socialista fue la solución perfecta pero no la más idónea. Cuando en la década de 1970 se produjo el baby boom, las nuevas construcciones fueron iguales que las de la década de 1950 convirtiendo a los apartamentos en "pequeñas ratoneras". Hay que decir que el problema persiste hasta la actualidad.

\subsection{El Estado de Bienestar durante la transición y consolidación de la democracia: 1989-2004}

Considerando que la transición y consolidación de la democracia polaca abarcó cronológicamente desde 1989 hasta el año 2004, -año en el que la República de Polonia adquirió la condición de Estado miembro de la Unión Europa-, las transformaciones políticas y socioeconómicas que se produjeron durante todo este periodo tuvieron como fin transformar el régimen comunista en un sistema de democracia parlamentaria y de economía de mercado.

Obsta decir que dicha transformación resultó ser un proceso arduo. A día de hoy, Polonia todavía no ha podido incorporarse a la zona euro a pesar del gran esfuerzo que han realizando los distintos gobiernos que se han sucedido en el poder para conseguirlo.

Respecto al objeto de estudio del presente artículo, el Estado de Bienestar, la evolución del mismo irá al compás de la propia evolución del Estado como ente jurídico-político pero también como garante de determinados derechos sociales de la ciudadanía.

Jacek Kuron, activista político durante la pretransición y ministro de Trabajo y Política Social durante el primer gobierno de Polonia como República Popular, debe ser considerado el alma mater de las reformas sociales que se llevarán a cabo durante los primeros años de esta nueva etapa. No obstante, dichas reformas no hubiesen surtido efecto sino hubiesen quedado encuadradas dentro de que lo que podríamos denominar como la "gran reforma" llevada a cabo por Leszek Balcerowicz ${ }^{3}$, ministro de Economía durante el gobierno de Tadeusz Mazowiecki.

3 El paquete de leyes destinado regular la economía fue presentado por Balcerowicz en diciembre de 1989. Tenía un carácter básicamente monetarista y comprendía un abandono radical de las reglas para dirigir la economía. El saneamiento de las finanzas y el equilibrio del presupuesto tenían que acompañar la privatización y la economía de mercado. El proyecto contó con el apoyo del Fondo Monetario Internacional, gracias al cual fue creado el Fondo de Estabilidad con un monto de 1000 millones de dólares. Este "seguro" para las reformas resultó, sin embargo, innecesario (Kieniewicz 2000: 306). 
Las primeras medidas económicas adoptadas para transformar la economía planificada hacia una economía de mercado tuvieron como principal consecuencia el aumento de los desempleados, aumento que se contrarrestó con la adopción de determinadas medidas sociales como las prejubilaciones o las pensiones por incapacidad, medidas que a la larga resultaron ser un estorbo para las reformas. De este modo, se consiguió mitigar el impacto inicial a pesar de que la creación de puestos de trabajo como objetivo deseable por un sector amplio de la población no fue considerado en ningún caso un objetivo prioritario por parte de los dos primeros gobiernos de la transición.

Puede decirse que desde 1989 hasta 1993 la característica principal en la transformación mencionada al comienzo del presente párrafo fue la crisis generalizada del sector económico redundado en una mínima protección de los derechos sociales. Las huelgas y las protestas en las calles mostraron una ciudadanía muy descontenta con las promesas realizadas por los dirigentes políticos, promesas que alcanzaban una expectativa de mejora en el nivel de vida de la población en su conjunto. Cuestiones como la reprivatización o la manumisión general, - entendida como el otorgamiento de los derechos de propiedad a todos los ciudadanos sobre las posesiones confiscadas durante la etapa comunista- no pudieron ser realizadas.

En una siguiente etapa que abarcaría desde 1993 hasta 1997, la mejora en los indicadores macro y microeconómicos redundó en una mejora de la política social. El respaldo jurídico a dicha política fue la promulgación de la nueva Constitución. El texto fue autorizado por la Asamblea Nacional el 2 de abril de 1997 y entró en vigor el 17 de septiembre. La ley fundamental definía a Polonia como un Estado democrático de derecho, en el que el poder pertenecía a la nación. Entre las garantías constitucionales se encontraban los derechos sociales. Fue durante los años mencionados cuando los paquetes de medidas gubernamentales adoptados en previsión del texto constitucional y que incidieron de lleno en derechos básicos como la vivienda, la educación y la sanidad, hicieron posible la transformación de todas aquellas instituciones garantes del Estado de bienestar dentro de la transformación profunda de la economía polaca desde un sistema planificado hacia el sistema de libre mercado.

En 1998, el gobierno liberal de Jerzy Buzek se comprometió a acelerar la privatización y descentralización económica con el fin de que Polonia pudiera, en un futuro no muy lejano, integrarse en la Unión Europea. Dicha descentralización económica afectó de lleno a la política social, a saber: se reorganizó la educación, se introdujo un sistema de seguros sobre la salud y se estableció un nuevo sistema de jubilación.

Hay que resaltar que los índices positivos que alcanzó Polonia en los principales indicadores económicos desde 1998 hasta 2003, estuvo relacionado con la estabilización de la posición del país en Europa. Después de ser admitida en 1995 en la 
Organización Mundial de Comercio (en adelante, OMC) y en 1996 en la Organización para la Cooperación y Desarrollo en Europa (en adelante OCDE), las autoridades mundiales del sector bancario mejoraron la evaluación de Polonia, lo que favoreció la continuidad de las inversiones, redundado en una mejora de la economía interna del país. De este modo, el Estado de Bienestar se fue transformado al compás de las propias transformaciones jurídico-políticas y socioeconómicas del país aunque de manera muy desigual.

Durante el periodo 1999-2000 las reformas que se hicieron en el servicio de salud pública, el sistema educativo y el sistema de pensiones tuvieron un efecto muy negativo en la sociedad. La frustración social contrastó con el éxito económico pero éste no fue suficiente para que el gobierno de coalición existente se mantuviera en el poder. En las elecciones presidenciales del 2000 Aleksander Kwasniewski fue reelegido como Presidente de la República de Polonia. Un año más tarde en las elecciones parlamentarias Leszek Miller saldría elegido Primer Ministro. Ambos líderes de la izquierda polaca, su objetivo en política exterior -así como del resto de los diferentes representantes de los partidos parlamentarios que conformaban el Sejm ${ }^{4}$, se centró en la aspiración de que Polonia ingresara como Estado miembro en la Unión Europea, cuya ampliación estaba prevista para el 2004.

La materialización de este objetivo común conllevó nuevos cambios económicos y sociales en el país del águila blanca. Puede decirse que, mientras Polonia completaba el tránsito de la economía planificada a la economía de mercado, la legislación europea le marcaba nuevos criterios políticos y económicos, criterios encuadrados en las condiciones de adhesión.

Así como Polonia necesitaba en el terreno económico un "gran empujón" para poder ingresar en la Unión Europea tal y como le ocurrió a España en su momento, la dimensión social de la integración del país del águila blanca, -entendida como la capacidad de la sociedad polaca para una plena interacción con las sociedades de otros países miembros-, estaba un poco más avanzada teniendo en cuenta como lo hace magníficamente Izabela Barlinska el estudio de los dos indicadores del capital humano de la sociedad polaca que pueden tener mayor relevancia a la hora de estudiar la integración social de Polonia en la Unión Europea, a saber: la educación y la salud, indicadores esenciales para entender la política social en la que quedan encuadrados los mismos y, por ende, el Estado de Bienestar.

En este sentido, y siguiendo a la citada autora, respecto al primero de los indicadores señalados, la educación, Polonia en comparación con otros países de la Unión Europea no se alejaba demasiado del porcentaje de personas que tenían una edu-

\footnotetext{
4 El Sejm hace referencia a la Cámara Baja del Parlamento polaco. Está compuesto por 460 miembros elegidos cada cuatro años.
} 
cación de nivel medio. De hecho, en la década de los años 90 Polonia se acercaba mucho a España e Italia, e incluso estaba por encima del porcentaje de Portugal, (Barlinska 2005: 29). Así, durante la oleada de inmigrantes polacos en la década de 1990 hacia países como España se constató la buena integración de los inmigrantes polacos en dicho país, debido, entre otras razones, al alto nivel respecto a la formación y educación y a su capacitación profesional. Aun así, hasta la entrada de Polonia en la Unión Europa el inmigrante polaco pasó de ser un refugiado político durante la salida de la población polaca en la etapa comunista a un "inmigrante económico" para llegar a convertirse en la actualidad en un ciudadano comunitario. (González y Merino 2005: 137).

No se puede decir lo mismo del segundo de los indicadores mencionados, la salud. Todavía durante la década de 1990, Polonia arrastraba un índice elevado de mortalidad infantil y de diferentes enfermedades como las cardiacas. La mala alimentación, el elevado consumo de alcohol o el estrés, fruto del sometimiento de la población a las transformaciones del sistema político y económico desde el año 1989 , redujeron considerablemente la esperanza de vida de la población polaca. Hay que recordar que las transformaciones del sistema económico realizadas por los sucesivos gobiernos del poder durante la década mencionada resultaron ser respecto a la educación, la vivienda y la salud insuficientes. Puede afirmarse que el Estado de Bienestar considerando los indicadores mencionados fue desarrollándose durante todos estos años en un nivel inferior.

\section{La integración de Polonia en la Unión Europea: luces y sombras del Estado del Bienestar}

La integración de Polonia en la Unión Europea supuso el punto de partida para el reconocimiento de Polonia como un Estado con una democracia parlamentaria ya consolidada y una economía de mercado en marcha. Pero Polonia siempre se ha sentido integrante de la "vieja Europa". Su milenaria historia política marcada por las sucesivas monarquías, su fuerte identidad nacional anclada en la religión cristiana y su preservación de su cultura aún en las peores etapas, ya sea cuando desapareció como Estado en tres ocasiones o cuando su soberanía quedo "secuestrada" durante el régimen comunista, han hecho del país del águila blanca un Estado fuertemente europeo.

Dicho lo anterior, no es menos cierto que, la integración de Polonia en organizaciones internacionales de carácter regional y mundial como la OTAN, la OCDE, el Consejo de Europa o la Unión Europa ha revestido al Estado polaco de un status jurídico-político "superior" del que carecía hasta hace unas décadas. Dicho status ha supuesto para Polonia un gran reto político y socioeconómico en el que todavía se encuentra inmerso. Como se señalaba al comienzo del presente artículo, Polonia no ha podido todavía integrarse en la zona euro pero las previsiones no son muy pesi- 
mitas siendo uno de los países del este de Europa que presentan una coyuntura económica favorable con una previsión de crecimiento para el 2013 estimada por la Comisión Europea en un 2,5\%.

No cabe duda de que la integración de Polonia en la Unión Europea en mayo de 2004 ha supuesto un avance cuantitativo y cualitativo en temas tan delicados como los que nos ocupan, a saber: política social, vivienda y salud. Las sucesivas regulaciones jurídicas incluidas en los recientes Tratados de Lisboa y el Tratado de Funcionamiento de la Unión Europea (en adelante, TFUE) junto con los ya existentes ha abierto el abanico normativo y las posibilidades de desarrollo práctico dentro del territorio nacional teniendo en cuenta que las materias mencionadas son competencia de los Estados miembro, en este caso del Estado polaco.

En este sentido hay que destacar el llamado Método de Coordinación Abierto, (en adelante MAC) creado en el marco de la política de empleo y el proceso de Luxemburgo definiéndose como un instrumento de la estrategia de Lisboa (2000). EI MAC proporciona un nuevo marco de cooperación entre los Estados miembros, con el objeto de lograr la convergencia entre las políticas nacionales para lograr objetivos comunes. En el marco de este método intergubernamental, los Estado miembro evalúan a otros a través del denominado "control de grupo". El MAC se aplica a los ámbitos que son competencia de los Estado miembro como el empleo, la protección social, la juventud, la educación y la formación.

El Estado polaco participa en el MAC desde el año 2005 con diversos programas nacionales como el elaborado en agosto de 2005, el llamado "Plan Nacional de Acción Pensión" con el fin de contribuir al diseño de unas directrices generales para la elaboración de una política común de pensiones para todo el territorio de la Unión Europea. Anteriormente al citado Plan, Polonia presentó otros programas. Cabe destacar la llamada "Estrategia Nacional para la mejora del empleo y el desarrollo de los recursos humanos 2000-2006", formulado en 1999 y considerado como un ejercicio práctico de cara a su presentación formal ante la Comisión más que un programa en sí mismo.

Junto a los ya citados, debe mencionarse el "Plan Nacional de Acción Empleo", aprobado en 2004 cuyo desarrollo sería para un año, es decir, hasta el 2005. El programa Ilamado "Estrategia Nacional para la inclusión social" aprobado en el año 2004 y cuyo objetivo prioritario se basó en el fomento del empleo en personas con riesgo de exclusión social. Dicho plan se prolongaría en el tiempo hasta el año 2010 ejecutándose mediante planes bianuales. Los tres últimos planes mencionados forman parte de un marco de actuación más amplio englobado en los llamados "Planes Nacionales de Acción para la protección social y la inclusión social" que abarcarían desde el año 2004 hasta el 2010.

Todos los programas mencionados tratan de recoger la nueva filosofía política inspiradora de la "Europa de los 27" en los ámbitos ya mencionados con anteriori- 
dad. El problema radica en que dichos programas son percibidos por la sociedad de manera desigual seguramente porque las medidas prácticas adoptadas posteriormente por los dirigentes políticos parecen no tener relación directa con lo redactado en cada uno de los planes. En el caso de Polonia, el actual primer ministro, Donald Tusk ${ }^{5}$ se presenta en la Unión Europea como un fuerte defensor y admirador de la misma y así es percibido por sus colegas europeos, haciendo ver que el Estado polaco esta realizando bien las tareas.

Cuestión bien distinta y mucho más compleja es la que hace referencia a la política interna del país. El panorama es poco halagüeño. Respecto al Estado de Bienestar, objeto de estudio en el presente artículo, Tusk ha puesto en marcha una serie de reformas sensibles como la del sistema de salud, la prescripción de fármacos y la reforma del sistema de pensiones, reformas que afectan de lleno al desarrollo de aquel en Polonia y su equiparación al resto de modelos existentes en Europa. De todos modos, es cierto que las diferentes organizaciones y sociales existentes, y también el propio gobierno es cada vez más consciente de las nuevas corrientes inspiradores de la política social como la llamada "economía social" proveniente de Francia y asumida por la Comisión Europea.

Mediante la misma, se trata de poner en evidencia el funcionamiento de unidades económicas que no tenga una finalidad estrictamente comercial pero que queden conectadas a las empresas para mejorar, por ejemplo, la inclusión laboral de personas con discapacidad física o intelectual entre otros colectivos de riesgo. El gobierno polaco, a través de la aprobación de diferentes leyes al respecto ha hecho posible que los gobiernos locales hayan puesto en marcha diferentes centros de integración para facilitar el empleo a personas discapacitadas y a personas que han sufrido problemas de alcoholismo o drogadicción.

Como conclusión final se podría decir que todas estas medidas y las que se vayan surgiendo en el futuro, son una muestra clara del avance del Estado de Bienestar en Polonia, avance que en ocasiones, se ve oscurecido por las decisiones políticas del gobierno de turno. Pero queda claro, que la integración de Polonia en la Unión Europea ha servido no sólo para revivir el debate "ser europeo" entre el pueblo polaco sino para inspirar un modelo europeo junto con los diferentes modelos nacionales en lo que al Estado de Bienestar se refiere como referente de un estadio superior: el Modelo Social Europeo. En el caso polaco, grupos de expertos y representantes de distintas organizaciones de ámbito sociopolítico junto con representantes gubernamentales llevan varios años debatiendo sobre cómo puede incardinarse dicho

5 DonaldTusk fue elegido como Primer Ministro en las elecciones legislativas del 2001, renovando con esta elección su mandato en el cargo. Fundó en el año 2001, junto con otros dos compañeros el partido llamado "Plataforma Cívica" con un ideario de centro-derecha basado en la defensa de ideas europeístas y democristianas. 
modelo en las sociedades de hoy día. Las preguntas que surgen no son pocas, a saber:

1. ¿Qué ventajas y desventajas presenta el Modelo Social Europeo en un mundo global y globalizado dominado por la competitividad?

2. ¿Qué es necesario cambiar pues en el modelo tradicional de Estado de Bienestar para alcanzar objetivos deseables para la mayoría de la población como un alto empleo, un sistema de asistencia sanitaria pública, unas pensiones dignas y una alta cohesión social entre otros temas?

Las respuestas se tornan complejas, sobre todo porque la dinámica económica en la que está inmersa Polonia desde su incorporación a la Unión Europea como Estado miembro en aras a alcanzar un determinado nivel de desarrollo económico que le equipare con el resto de sus socios comunitarios, ha servido en ocasiones de cortapisa al desarrollo social. Pero las demandas sociales son cada vez mayores. Los diferentes gobiernos polacos que se sucedan en el poder no pueden ni podrán hacer caso omiso de las mismas.

\section{Bibliografía}

Barlinska, I. 2005. "La integración de Polonia en la unión europea: indicadores sociales y datos de la opinión pública española". Pp. 29-35 en Polonia y España ante el futuro de la Unión Europea, coordinado por R. D. Torres Kumbrián, G. Bernatowicz y J. Grodzka. Madrid: Librería Popular.

Bauman, Z. 2007. Vida líquida. Barcelona: Paidós.

Engels, F. 1974. "Anti Dühring". Pp. 49-66 en La sociedad comunista, K. Marx, F. Engels y V. Lenin. Madrid: Akal.

Fernández García, T., López Pelaéz, A. 2006. “El Estado de Bienestar: orígenes y perspectivas". Pp. 15-47 en Política Social y Estado de Bienestar, coordinado por C. Alemán y T. Fernández. Valencia: Tirant lo Blanch.

Garcés, J. 2006. "Política social e ideología". Pp. 103-135 en Política Social y Estado de Bienestar, coordinado por C. Alemán y T. Fernández. Valencia: Tirant lo Blanch.

Golinowska, A. 2009. "The national model of the welfare state in Poland". Pp. 215-255 en Diversity and Commonality in European Social Policies: The Forging of a European Social Policy, coordinated by S. Golinowska, P. Hengstenberg y M. Zukowski. Warsaw: Friedrich- Ebert-Stiftung and Wydawnictwo Naukowe Scholar.

González Martínez, E., Merino, A. 2005. “El contexto inmigratorio de la España del fin del milenio: el caso polaco". Pp. 117-144 en España y Polonia: Ios encuentros, coordinado por E. González y M. Nalewajko. Madrid: Consejo Superior de Investigaciones Científicas.

Keynes, J.M. 1991. Las consecuencias económicas de la paz. Barcelona: Crítica.

Kieniewicz, J. 2001. Historia de Polonia. México: Fondo de Cultura Económica. 


\section{ARTICULOS/ARTICLES}

Empresas spin-off y género: diferencias entre hombres y mujeres en la creación de empresas de base tecnológica / Spin-off and gender: differences between men and women in the creation of technology-based companies

Ignasi Brunet Icart y Joan Rodríguez Soler

Custodia compartida: atribución vivienda familiar / Shared custody: the acquisition the family living Beatriz Escudero Berzal

Aproximación al estado de bienestar en Polonia / Aproximation to the welfare state in Poland Paloma Serrano Postigo

Menores víctimas de la violencia de género: propuesta de proyecto educativo / Minours who are victims of gender-violence. Proposal for an educational project

Luis Manuel Rodríguez Otero

La obesidad paradójica: construcción de una imagen corporal contradictoria / Paradoxical obesity: building a contradictory body image

Martha Leticia Cabello Garza y María Concepción Arroyo Rueda

\section{RESENAAS/REVIEWS}

Del Fresno García, M., Segado Sánchez-Cabezudo, S., López Peláez, A. (eds). Trabajo social con comunidades en el siglo XXI / Social Work with communites in the XXI Century (por Yolanda Meneses García)

Sagrario Segado Sánchez-Cabezudo, Miguel del Fresno García y Antonio López Peláez (eds.). Modelos de Trabajo Social con grupos: nuevas perspectivas y nuevos contextos / Models of social work with groups: new perspectives and new contexs (por Emilio Díaz de Mera)

Antonio López Peláez (ed.) The Robotics Divide: a New Frontier in the 21st Century? / La brecha robótica: ¿una nueva frontera en el siglo XXI? (por Raquel Pérez García)..... 